\title{
Pleth variability index and fluid management practices: a multicenter service evaluation
}

\author{
Patrice Forget ${ }^{1 *}$ (D), Simon Lacroix ${ }^{2}$, Eric P. Deflandre ${ }^{2,3,4,5}$, Anne Pirson ${ }^{6}$, Nicolas Hustinx ${ }^{6}$, Olivier Simonet ${ }^{7}$, \\ Fabrice Wandji ${ }^{7}$, Serge von Montigny ${ }^{8}$ and Jibba Amraoui ${ }^{9}$
}

\begin{abstract}
Objectives: The introduction of a new technology has the potential to modify clinical practices, especially if easy to use, reliable and non-invasive. This observational before/after multicenter service evaluation compares fluid management practices during surgery (with fluids volumes as primary outcome), and clinical outcomes (secondary outcomes) before and after the introduction of the Pleth Variability Index (PVI), a non-invasive fluid responsiveness monitoring.

Results: In five centers, 23 anesthesiologists participated during a 2-years period. Eighty-eight procedures were included. Median fluid volumes infused during surgery were similar before and after PVI introduction (respectively, $1000 \mathrm{ml}$ [interquartile range 25-75 [750-1700] and $1000 \mathrm{ml}$ [750-2000]). The follow-up was complete for 60 from these and outcomes were similar. No detectable change in the fluid management was observed after the introduction of a new technology in low to moderate risk surgery. These results suggest that the introduction of a new technology should be associated with an implementation strategy if it is intended to be associated with changes in clinical practice.
\end{abstract}

\section{Introduction}

The management of intraoperative blood volume and the major complications of both hypovolemia and hypervolemia remain major and unresolved issues [1]. It has been suggested that dynamic parameters such as pulse pressure or stroke volume variation may, at least partially, solve this problem [2]. Dynamic parameters assess hemodynamic response to respiratory variations which allows to monitor the fluid-dependency status. They have been well validated for the prediction of the response to a fluid challenge [2]. The Pleth Variability Index (PVI), as

*Correspondence: forgetpatrice@yahoo.fr

${ }^{1}$ Institute of Applied Health Sciences, Epidemiology group, School

of Medicine, Medical Sciences and Nutrition, University of Aberdeen, NHS

Grampian, Department of Anaesthesia, Aberdeen, UK

Full list of author information is available at the end of the article a dynamic and non-invasive parameter, could fill a gap for the low to moderate risk patient and procedure, especially when cardiac output monitoring is not considered [2]. To improve fluid management, the optimization of the PVI value has been proposed $[3,4]$ by targeting a PVI value between 10 and $13 \%$. Optimizing this value may help optimize cardiac output without taking the risk of giving too much fluids (i.e. fluids not associated with a significant increase in cardiac output) (Fig. 1). However, the current ability of the introduction of PVI on its own to change the practice of clinicians is not known.

This multicenter service evaluation aims to establish whether the introduction of the PVI in the clinical practice is associated with a modification of the practice during low to moderate risk surgery. 


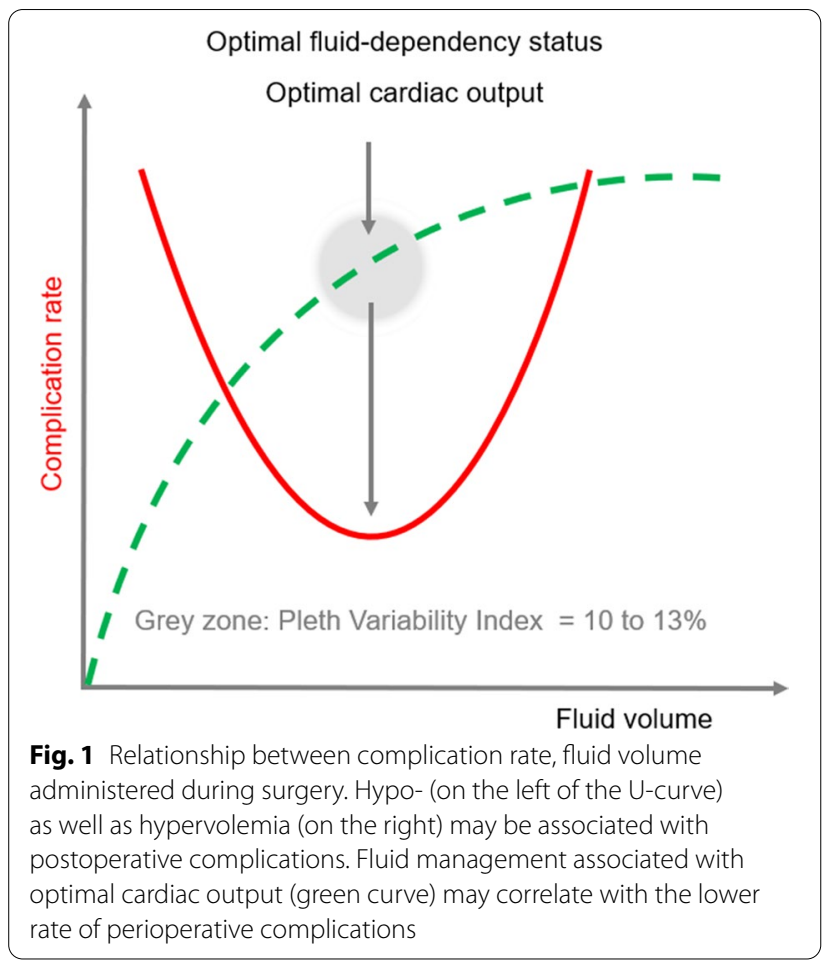

\section{Main text}

\section{Subjects and methods}

\section{Design and outcomes}

This before/after multicenter service evaluation compares the fluid volumes used before and after the introduction of the PVI, focusing on fluids volumes (primary outcome), with clinical outcomes as quality indicators (secondary outcomes).

Thus, three consecutive phases were planned: a pilot survey (preparatory phase), a phase 1 (before the introduction of the PVI) and a phase 2 (after).

This work is presented following the Strengthening the Reporting of Observational Studies in Epidemiology (STROBE) Statement for cohort studies [5].

\section{Ethics committee consideration}

This multicenter service evaluation aims to assess how clinicians change their fluid management after the introduction of the PVI in three kind of surgery: Knee/hip arthroplasty, colorectal surgery. Written informed consent was waived by the ethics committee of the principal investigator (Patrice Forget, affiliated to the UCLouvain in 2012) (26th March 2012, Chairperson: Prof. J-M Maloteaux, Commission d'Ethique Biomédicale Hospitalo- Facultaire, Institution: UCLouvain), because considered as a practices audit, the essential information to be recorded being physicians' practices changes, without any breach in patient confidentiality. The project was registered before any data collection (Clinicaltrials.gov: NCT02271841) and performed in accordance with the ethical standards of the Declaration of Helsinki (1964) and its subsequent amendments.

\section{Settings}

The recruitment of a minimum of 3 centers was anticipated in Belgium and/or France who had no significant experience with the technology, to reach approximately 30 participating anesthesiologists. All the centers $(n=10)$ that worked within the network of the university hospital were considered.

\section{Inclusion / exclusion criteria}

Fives sites were recruited to participate in this research; each site may be in use of Masimo technology, but not PVI at the time. More than one anesthesiologist per center was recommended. Into these centers, all anesthesiologists had the opportunity to participate.

The project was limited to surgical procedures like knee/hip arthroplasties and colorectal surgery. To avoid a too high heterogeneity challenging data interpretation, patients under 18 years and ASA (American Society of Anesthesiologists) 4 were excluded.

\section{Equipment}

Standard of care monitoring for surgery included a pulsoxymeter, continuous electrocardiogram, non-invasive blood pressure monitoring, gas analyzers including capnography. No limitation was suggested in the use of other type of monitoring.

The use of Masimo Radical 7 devices SET technology with PVI feature was possible after the introduction of the technology, which was preceded by an appropriate training (delivered by PF, proposing a grey zone approach for the decision of fluid loading above a PVI value of more than 10 to $13 \%$ during more than $5 \mathrm{~min}$ ) $[3,4]$. During this training, the determinants of cardiac output and the influence of intravascular blood volume were discussed. The advantages and disadvantages of the different types of advanced hemodynamic monitoring were presented and discussed with the participants. The information was based on a previously published expert consensus [2].

\section{Statistical analysis}

Based on previously published data in similar procedures, with low expected variability, a difference of $250 \mathrm{ml} \pm 250 \mathrm{ml}$ could be anticipated [4]. This small difference was chosen to unmask any practice change, small or large in the fluid management, independently on any clinical significance. The type of surgery was considered for low variability, rather than being able to measure 
improvement in outcome. The clinical results were considered as secondary outcomes, forming part of a service evaluation. For this, we used the Dindo-Clavien and the Postoperative Morbidity Survey classifications [6, 7].

A sample size calculation showed that at least 22 procedures recorded before and after (44 in total) would be sufficient to exclude the null hypothesis (being the absence of a difference of $250 \mathrm{ml} \pm 250 \mathrm{ml}$ ) with an alpha of 0.05 and a power of $90 \%$ [4]. We decided not to restrict the number of procedures, up to end 2018.

Data were collected and managed using REDCap (REsearch Electronic Data Capture) tools [8]. The forms were designed by the research team and tested with dummy data before use. Non-response bias was minimized as much as possible through automatic reminders and personal communication from the lead investigator. There was no incentive other than to receive the material for free and be part of the research project.

As normal distribution was rejected in most cases, Mann-Whitney U test was used for the comparisons. Data are given with mean \pm SD or median [interquartile range 25-75] [IQR25-75], and 95\% confidence interval $(95 \% \mathrm{CI})$ as appropriate. A p-value of less than 0.05 was considered significant. No subgroup analysis, sensitivity nor other inferential analysis was planned. Statistica version 7.0 (STATSOFT, Tusla, USA) was used for all the analyses.

\section{Results}

\section{Pilot survey}

A pilot survey was sent to 19 anesthesiologists to document the current practices (Additional file 1: Tables S1, $\mathrm{S} 2$, Figure S1). These nineteen were all from the 23 ultimately included who accepted to respond to this pilot questionnaire. This confirmed that an advanced and/or invasive monitoring was frequently used by most of the practitioners.

\section{Participants}

After the pilot survey, 75 anesthesiologists were approached, in five centers, by the local investigator and 23 completed the project (Additional file 1: Fig. S1). The reasons for not including the others were not documented. These practitioners progressively started the first phase (before the introduction of the new monitoring), including consecutive patients from February 2015 up to October 2017. After the introduction of the PVI, the second phase of the data collection occurred for all the centers during the year 2018.

\section{Patients and procedures}

In the five centers, 88 patients and procedures were followed (Table 1). The follow-up was complete for 60 patients from the 88 . Ventilation practices were slightly different (more frequently administered lower tidal volumes) but this was not statistically significant (Table 1).

The total volume administered during surgery was similar when comparing the period before PVI introduction with the period after (primary outcome) (median [IQR25-75]: respectively, $1000 \mathrm{ml} \mathrm{[750-1700]} \mathrm{and}$ $1000 \mathrm{ml}[750-2000])$. The other outcomes were also similar (Table 2, Additional file 1: Figure S2) ( $>0.05$ for all the comparisons).

\section{Discussion}

No detectable change occurred before and after the introduction of the PVI. Particularly, no change was observed in the average amounts of fluids used during prosthesis and colonic surgeries after this introduction of a fluid responsiveness monitoring. However, this does not permit to exclude any change in the practice that may occur in particular patients, but not visible in aggregated analyses.

Moreover, the changes in practices may be limited by several factors, notably a probable evolution in the ventilatory practices, toward lower tidal volumes and slightly higher frequency, even if not statistically significant. Interestingly, the pilot survey showed that most of the practitioners declared that the institution does not have any written protocol, care guide or statement concerning hemodynamic management. This may introduce the question whether a new monitoring may help if not introduced in a protocol. An observation of this is the fact that, in a meta-analysis on goal-directed therapy (GDT), the effect of the intervention was particularly evident in older series, and namely in non-ERAS (enhanced recovery after surgery) programs [8]. One may speculate that the existence of a protocol (in this case, ERAS program) may have an important impact interacting with the added value of a new monitoring. These patients may also present a lower risk profile, and then benefiting less of a GDT. On the other hand, the introduction of a quality improvement program has been recently shown as complex, and not necessarily directly associated with improved outcomes [9]. The relative low introduction rate of the cardiac-output monitoring in the $\mathrm{EPOCH}$ trial reinforced our observation that invasive technologies would not be seen suitable in most low to moderate risk procedures [1]. 
Table 1 Characteristics of the 88 patients and procedures

\begin{tabular}{|c|c|c|c|c|c|c|}
\hline & Valid N & Mean & Std.Dev & Median & Lower quartile & Upper quartile \\
\hline Age (years) & 88 & 68.5 & 9.5 & 68 & 63 & 75 \\
\hline Before & 54 & 69.2 & 9.1 & 68 & 64 & 75 \\
\hline After & 34 & 67.3 & 10.1 & 68 & 62 & 73 \\
\hline Sex: males; females & $35 ; 53$ & & & & & \\
\hline Before & $21 ; 33$ & & & & & \\
\hline After & $13 ; 21$ & & & & & \\
\hline Height (cm) & 86 & 165.4 & 9.9 & 165 & 158 & 172 \\
\hline Before & 52 & 164.9 & 10.2 & 165 & 157 & 172 \\
\hline After & 34 & 166.1 & 9.7 & 165 & 160 & 175 \\
\hline Weight (kg) & 87 & 77.4 & 18.7 & 76 & 60 & 94 \\
\hline Before & 53 & 77.8 & 20.8 & 77 & 60 & 95 \\
\hline After & 34 & 76.8 & 15.2 & 76 & 67 & 84 \\
\hline ASA: $1 ; 2 ; 3 ; 4$ (n) & $7 ; 56 ; 16 ; 0(/ 79$ & & & & & \\
\hline Before & $5 ; 33 ; 7 ; 0(/ 45)$ & & & & & \\
\hline After & $2 ; 23 ; 9 ; 0(/ 34)$ & & & & & \\
\hline Length of the surgery (min) & 85 & 136 & 76 & 120 & 90 & 180 \\
\hline Before & 52 & 127 & 66 & 103 & 90 & 150 \\
\hline After & 33 & 149 & 90 & 125 & 90 & 195 \\
\hline General anesthesia & $62(/ 88)$ & & & & & \\
\hline Before & $43(/ 54)$ & & & & & \\
\hline After & $24(/ 34)$ & & & & & \\
\hline TKP; THP; Colonic surgery & $27 ; 31 ; 30(/ 88)$ & & & & & \\
\hline Before & $17 ; 22 ; 15(/ 54)$ & & & & & \\
\hline After & $10 ; 9 ; 15(/ 34)$ & & & & & \\
\hline Laparoscopic approach (n) & 29 & & & & & \\
\hline Before & 14 & & & & & \\
\hline After & 15 & & & & & \\
\hline Tidal volume (ml) & 62 & 524 & 274 & 460 & 450 & 500 \\
\hline Before & 39 & 559 & 338 & 500 & 450 & 525 \\
\hline After & 23 & 464 & 70 & 450 & 400 & 475 \\
\hline Frequency at beginning (cpm) & 63 & 14 & 2 & 14 & 12 & 14 \\
\hline Before & 40 & 13 & 2 & 13 & 12 & 14 \\
\hline After & 23 & 15 & 3 & 14 & 12 & 16 \\
\hline
\end{tabular}

ASA American Society of Anesthesiology score, TKP Total knee prosthesis placement, THP Total hip prosthesis placement, Frequency at the beginning: refers to respiratory frequency at the beginning of the surgery, cpm cycles per minute

The question remains whether a non-invasive technology may replace more invasive ones for low to moderate risk surgery. This study was not designed for, but the potential exists and would merit to be further explored. Exploratory analyses showed that $38 / 54$ procedures $(70 \%)$ before the introduction of the PVI did not reported any use of an invasive monitoring vs. 27/34 (79\%) ( $>>0.05)$ suggesting a shift toward less invasive monitoring.

In conclusion, this work shows that no detectable change in the fluid management was observed after the introduction of a new technology in low to moderate risk surgery.

\section{Limitations}

The limitations of this work are linked to the small sample size, small tidal volumes, laparoscopic procedures, low risk surgery, and the lack of generalizability in other contexts, like in higher risk surgery, but highlight the fact that even in low variability procedures, there is a need for implementation of protocols and a place for non-invasive monitoring. 
Table 2 Outcomes of the 88 patients and procedures

\begin{tabular}{|c|c|c|c|c|}
\hline & Valid N & Median & Lower quartile & Upper quartile \\
\hline \multicolumn{5}{|l|}{ Primary outcome } \\
\hline IV volume administered (ml) & 88 & 1000 & 750 & 1750 \\
\hline Before & 54 & 1000 & 750 & 1700 \\
\hline After & 34 & 1000 & 750 & 2000 \\
\hline \multicolumn{5}{|l|}{ Secondary outcomes } \\
\hline Length of stay, ICU (days) & 60 & 0.0 & 0.0 & 0.0 \\
\hline Before & 35 & 0.0 & 0.0 & 0.0 \\
\hline After & 25 & 0.0 & 0.0 & 0.0 \\
\hline Length of hospital stay (days) & 60 & 5.0 & 4.0 & 6.0 \\
\hline Before & 35 & 5.0 & 4.0 & 7.0 \\
\hline After & 25 & 5.0 & 4.0 & 6.0 \\
\hline \multicolumn{5}{|c|}{ Postop. complications (Dindo-Clavien) } \\
\hline (Grade $0 ; 1 ; 2 ; 3 ; 4 ; 5)$ & 60 & $38 ; 17 ; 3 ; 2 ; 0 ; 0$ & & \\
\hline Before & 35 & $21 ; 9 ; 3 ; 2 ; 0 ; 0$ & & \\
\hline After & 25 & $17 ; 8 ; 0 ; 0 ; 0 ; 0$ & & \\
\hline
\end{tabular}

IV: intravenous; ICU: Intensive care unit; Dindo-Clavien classification of postoperative complications: Grade 0: no complication. Grade I: Any deviation from the normal postoperative course without the need for pharmacological treatment or surgical, endoscopic and radiological interventions. Allowed therapeutic regimens are: drugs as antiemetics, antipyretics, analgesics, diuretics and electrolytes and physiotherapy. This grade also includes wound infections opened at the bedside. Grade II: Requiring pharmacological treatment with drugs other than such allowed for grade I complications. Blood transfusions and total parenteral nutrition are also included. Grade III: Requiring surgical, endoscopic or radiological intervention. Grade IV: Life-threatening complication. Grade V: Death of a patient

\section{Abbreviations}

PVI: Pleth Variability Index; STROBE: Strengthening the Reporting of Observational Studies in Epidemiology; ASA: American Society of Anesthesiologists; REDCap: REsearch Electronic Data Capture; SD: Standard deviation; IQR: Interquartile range; Cl: Confidence interval; GDT: Goal-directed therapy; ERAS: Enhanced recovery after surgery; EPOCH trial: Enhanced Peri-Operative Care for High-risk patients trial.

\section{Supplementary Information}

The online version contains supplementary material available at https://doi. org/10.1186/s13104-021-05705-6.

Additional file 1: Table S1. Background of 19 anesthesiologists responding to a pilot survey regarding fluid management. IQR: interquartile range. Table S2. Practices of 19 anesthesiologists responding to a pilot survey regarding goal directed therapy (GDT) for fluid management. Figure S1. Flow chart. Figure S2. Total volume administered in 88 patients before and after the introduction of the Pleth Variability Index (expressed as median, 25-75 interquartile range and range). IV: intravenous.

\section{Acknowledgements}

None

\section{Authors' contributions}

PF designed the work; PF, SL, EPD, AP, NH, OS, FW. SVM and JA contributed to the data collection, the discussions, commented the manuscript and approved the final version. All authors read and approved the final manuscript.

\section{Funding}

Masimo Co. provided support for data capture materials, devices and sensors. Masimo Co. had no role in the design of the study and collection, analysis, and interpretation of data and in writing the manuscript.

\section{Availability of data and materials}

The data are available upon reasonable request addressed to the corresponding author (Patrice Forget, forgetpatrice@yahoo.fr).

\section{Declarations}

\section{Ethics approval and consent to participate}

The study was submitted to and approved by the institutional ethics committee. The need for formal ethics approval was waived by this committee and written informed consent was waived (26th March 2012, Chairperson: Prof. J-M Maloteaux, Commission d'Ethique Biomédicale Hospitalo- Facultaire, Institution: UCLouvain), because considered as a practices survey, the essential information to be recorded being physicians' practices changes, and not specifically patient information. Registered on Clinicaltrials.gov: NCT02271841.

\section{Consent for publication}

Not applicable.

\section{Competing interests}

Patrice Forget has been member of advisory boards of Masimo Co. All the other authors have no competing interest.

\section{Author details}

${ }^{1}$ Institute of Applied Health Sciences, Epidemiology group, School of Medicine, Medical Sciences and Nutrition, University of Aberdeen, NHS Grampian, Department of Anaesthesia, Aberdeen, UK. ${ }^{2}$ Clinique Saint-Luc of Bouge, Department of Anaesthesia, Extracorporeal Circulation Team, Namur, Belgium. ${ }^{3}$ Clinique Saint-Luc of Bouge, Department of Anaesthesia, Namur, Belgium. ${ }^{4}$ Cabinet Medical ASTES, Jambes, Belgium. ${ }^{5}$ University of Liege, Liege, Belgium. ${ }^{6}$ Grand Hopital de Charleroi, Charleroi, Belgium. ${ }^{7}$ Centre Hospitalier de Wallonie Picarde, Department of Anaesthesia, Tournai, Belgium. ${ }^{8} \mathrm{Centre} \mathrm{Hospitalier}$ Mons-Warquignies, Department of Anaesthesia, Mons, Belgium. ${ }^{9}$ Institut du Cancer, Montpellier, France. 
Received: 16 March 2021 Accepted: 19 July 2021

Published online: 28 July 2021

\section{References}

1. Peden CJ, Stephens T, Martin G, Kahan BC, Thomson A, Rivett K, Wells D, Richardson G, Kerry S, Bion J, Pearse RM; Enhanced Peri-Operative Care for High-risk patients (EPOCH) trial group. Effectiveness of a national quality improvement programme to improve survival after emergency abdominal surgery (EPOCH): a stepped-wedge cluster-randomised trial. Lancet. 2019; S0140-6736(18)32521-2.

2. Vincent JL, Pelosi P, Pearse R, Payen D, Perel A, Hoeft A, Romagnoli S, Ranieri VM, Ichai C, Forget P, Rocca GD, Rhodes A. Perioperative cardiovascular monitoring of high-risk patients: a consensus of 12. Crit Care. 2015;19(1):224

3. Forget $\mathrm{P}$, Lois F, De Kock M. Does Pleth variability improves fluid management during major abdominal surgery? Anesth Analg. 2010;111(4):910-4.

4. Forget P, Lois F, Kartheuser A, Leonard D, Remue C, De Kock M. The concept of titration can be transposed to fluid management. But does is change the volumes? A randomised trial on Pleth variability index during fast-track colonic surgery. Curr Clin Pharmacol. 2013;8(2):110-4.

5. von Elm E, Altman DG, Egger M, Pocock SJ, Gotzsche PC, Vandenbroucke JP. The Strengthening the Reporting of Observational Studies in
Epidemiology (STROBE) Statement: guidelines for reporting observationa studies. PLOS Med. 2007;4(10):e296.

6. Grocott MP, Browne JP, Van der Meulen J, Matejowsky C, Mutch M, Hamilton MA, Levett DZ, Emberton M, Haddad FS, Mythen MG. The Postoperative Morbidity Survey was validated and used to describe morbidity after major surgery. J Clin Epidemiol. 2007;60(9):919-28.

7. Dindo D, Demartines N, Clavien PA. Classification of surgical complications: a new proposal with evaluation in a cohort of 6336 patients and results of a survey. Ann Surg. 2004:240(2):205-13.

8. Harris PA, Taylor R, Thielke R, Payne J, Gonzalez N, Conde JG. Research electronic data capture (REDCap) — a metadata-driven methodology and workflow process for providing translational research informatics support. J Biomed Inform. 2009;42(2):377-81.

9. Rollins KE, Lobo DN. Intraoperative goal-directed fluid therapy in elective major abdominal surgery: a meta-analysis of randomized controlled trials. Ann Surg. 2016:263(3):465-76.

\section{Publisher's Note}

Springer Nature remains neutral with regard to jurisdictional claims in published maps and institutional affiliations.
Ready to submit your research? Choose BMC and benefit from:

- fast, convenient online submission

- thorough peer review by experienced researchers in your field

- rapid publication on acceptance

- support for research data, including large and complex data types

- gold Open Access which fosters wider collaboration and increased citations

- maximum visibility for your research: over $100 \mathrm{M}$ website views per year

At BMC, research is always in progress.

Learn more biomedcentral.com/submissions 